\title{
Holistic Analysis of the Classroom
}

\author{
Mirko Raca \\ École polytechnique fédérale de Lausanne EPFL \\ RLC D1 740 (Rolex Learning Center), Station 20 \\ $\mathrm{CH}-1015$ Lausanne, Switzerland \\ mirko.raca@epfl.ch
}

\author{
Pierre Dillenbourg \\ École polytechnique fédérale de Lausanne EPFL \\ RLC D1 740 (Rolex Learning Center), Station 20 \\ $\mathrm{CH}-1015$ Lausanne, Switzerland \\ pierre.dillenbourg@epfl.ch
}

\begin{abstract}
Should we judge the quality of the class by the grades the students and teacher get at the end of the semester or how the group collaborated during the semester towards acquiring new knowledge? Up until recently, the later approach was all too inaccessible due to complexity and time needed to evaluate every class. With the development of new technologies in different branches of video processing, gaze tracking and audio analysis we are getting the opportunity to go further with our analysis and go around the potential problem substitution into which we were previously forced.

We present our efforts to record student-student and studentteacher interactions within a classroom eco-system. For this purpose, we developed a multi-camera system for observing teacher actions and students reactions throughout the class. We complemented the data with a mobile eye-tracker worn by the teacher, quantitative questionnaire data collection, as well as in-depth interviews with students about their impressions of the classes they took, and about our intervention. The seven-part experiment was conducted during the autumn semester of 2013 , in two classes with over 60 participants.

We present the conclusions we reached about the experiment format, visualize the preliminary results of our processing and discuss other options we are considering for our further experiments. We aim to explore further possibilities for analysing classroom life in order to create a more responsive environment to the needs of the students.
\end{abstract}

\section{Keywords}

video analysis, computer vision, tracking, intrusiveness, head pose, classroom

\section{INTRODUCTION}

Considering the socio-material [16] dichotomy of learning, in the recent years we saw an increase in analysis of digital footprints produced by the students, with the rationalization that it appropriately represents the sociological aspect

Permission to make digital or hard copies of all or part of this work for personal or classroom use is granted without fee provided that copies are not made or distributed for profit or commercial advantage and that copies bear this notice and the full citation on the first page. Copyrights for components of this work owned by others than the author(s) must be honored. Abstracting with credit is permitted. To copy otherwise, or republish, to post on servers or to redistribute to lists, requires prior specific permission and/or a fee. Request permissions from permissions@ acm.org. MLA'14, November 12, 2014, Istanbul, Turkey.

Copyright is held by the owner/author(s). Publication rights licensed to ACM. ACM 978-1-4503-0488-7/14/11 ...\$15.00.

http://dx.doi.org/10.1145/2666633.2666636. of the learning process. Current research attempts try to strike a balance between how natural is the digitalization which we impose on the learning process, and how detailed is the information we can capture about the learning process. In the case of distance learning or MOOCs (Massive On-line Open Courses) [11] where the interaction between the students and the content is governed by the digital distribution system, a central point of analysis naturally arises. However, in the still dominant form of education, the physical classrooms, the technology-mediated learning requires a lot more planing for effective integration. And if we need to impose technological aids just to track the students progress, do we have the justification to use them at all?

Digital signal processing in various forms has been present for the last 50 years. With the maturing of the techniques and cheaper consumer-level sensors, the benefits of developed audio analysis (speech recognition, speaker differentiation), video processing (face detection, person tracking, pose estimation), written material analysis (OCR, augmented reality) are trickling down from specialized areas into different usage scenarios. In the case of education, these techniques now allows us to capture the aspects of the learning experience such as classroom interaction [23], content generation [20], discussion dynamics [4][26] and others, while still remaining unobtrusive [29].

Unlike people, machines have far greater capacity for analysis, and can handle data of much higher resolution than that manageable by humans. This observation gave rise to the machine learning (ML) field which is based on the idea that high-complexity mathematical models can be as effective as human reasoning given sufficient training data. This is true for problems such as binary classification, categorization or regression, and as previously stated - algorithms can beat human assessment in terms of precision. But in case of social interactions, not even humans are completely aware what forms the "quality"[9]. In case of the classroom, we see no reason to trade a professional teacher with years of experiences for a modelled assessment of students performance, but we do wish to give the teachers a richer input on which to base their conclusions.

In order to explore salient indicators of human interaction, we conducted longitudinal experiment which was designed to collect as many facets of classroom life as we could without disrupting the learning experience. Our richest source of data is based on the idea of observing students' behavior with consumer-grade video cameras. We based the approach on the promise that the computer vision (CV) technologies have shown in the recent past in extracting highly informa- 
tive features. However, although we see $\mathrm{CV}$ as an excellent source of data, we needed to complement them with a set of more traditional measurements in order to assess and establish the foundations of their usefulness.

The effort of the experiment consisted of seven recorded classroom session, followed by in-class or post-class questionnaires and interviews. The recorded sessions collected video footage of the students' in-class actions, teachers' movement, and the material presented on the slides. We attempted 2 different types of questionnaires, designed for in-class and post-class sampling of student opinion, and complemented the questionnaires with interviews for further qualitative insights. With the agreement of the teachers, we also used mobile eye trackers in order to capture teachers' audio, firstperson video from the teachers' perspective and gaze information.

\section{RELATED WORK}

A number of elements shape our perception of learning our interest in the subject, teacher's approach, and learning environment consisting of both persons and objects. For a long time learning needed to be high-potent, given that the lecturer had a limited time to transfer knowledge to the students. This weight was lifted with the introduction of high-quality openly available courses (MOOCs) [11], in case of the highly-motivated students, at the same time emphasizing the importance of student motivation which was implicit in the obligatory education[7].

Systems for pooling student opinions already exist in the form of dedicated devices (e.g. clickers [6]), or smart-phone applications [24]. Our approach, the usage of cameras as base for an observational platform, is inspired by the ideas of unobtrusive measurements [29]. In the complex ecosystem such as a classroom environment, we feel that overloading the students or the teachers with additional distractions can hinder the learning process, rather than enhance it.

The analysis of non-verbal signals and especially gaze information has the potential to aid humans in a number of situations, but our focus lies in the application of students' behaviour analysis for the purpose of learning analytic. Experienced teachers have the habit of scanning the classroom for low attention students. Teachers as orchestrators of the learning process [12][13] go beyond simple presentation of teaching material. They take on themselves the responsibility to engage different groups of students and they need to constantly modify their approaches for maximal impact [25]. By providing the teachers with the tool which can analyze student engagement we delegate the objectivity of selfreflection, enabling teachers to step around potential psychological biases caused by classroom arrangement [10].

Other effects in group behaviour such as automatic crowd segmentation based on motion have been observed in spectator sports [8]. With the introduction of reliable pose estimations, explorations spread into body movement, with the cost of introducing a depth-camera capturing constraints [28]. In the learning domain, studies in motion tracking in the classroom [23][22] indicate that there are multiple measurements which can be potentially useful for teacher's performance and audience behavior analysis. Facial features have been explored before in single-user settings with significant success [14], and more complex multi-modal systems for emotional assessment are also known in the literature [3],

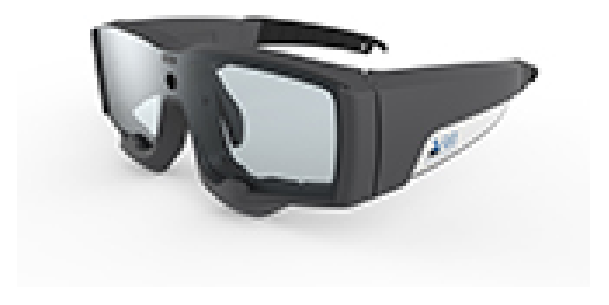

Figure 2: SMI Portable eye-tracker

although the cost of such systems puts those efforts more in the exploratory domain.

\section{METHOD}

We followed two groups of students over the total of seven lectures. The students were from the first year of master studies following two different subjects (each class was always held by the same person). Demographic statistics of the student participants are given in Table 1 . We used a multitude of measures and activities which we will now go over individually.

\subsection{Cameras and eye-tracker}

The main goal of the experiment was to record the behaviour of students during the class. To accomplish this, we installed a system of web-cameras around the black-board directed towards the student audience of the class. Several cameras (4-6) were needed in order to capture the entire class population.

In addition to the cameras observing the students, we had an additional camera in the back of the class capturing teacher's actions and slide changes. Even though the recorded video was in HD resolution (1920x1080 pixels), due to the distance from the teacher, only the major body movement and gesticulation were captured.

For capturing the teacher's perspective, we also used a mobile eye-tracking device (shown in Fig.2), which teachers wore during the entire lesson.

\subsection{Questionnaires}

In order to validate our observations from the video sequences, we distributed a questionnaire among the student audience of the classes. The questionnaire consisted of 4 Likert scales (1-10) capturing:

- estimation of student's own perceived attention,

- estimation of attention for the rest of the student population,

- perceived teacher's energy,

- presented material's importance to personal study (irrelevant - highly important).

In the original format of the experiment, the procedure of the experiment was based on strobe-sampling the information from the students. By using a sound signal, we interrupted the class at four random (equally spaced) times, and asked the students to fill out the questionnaire. In both 


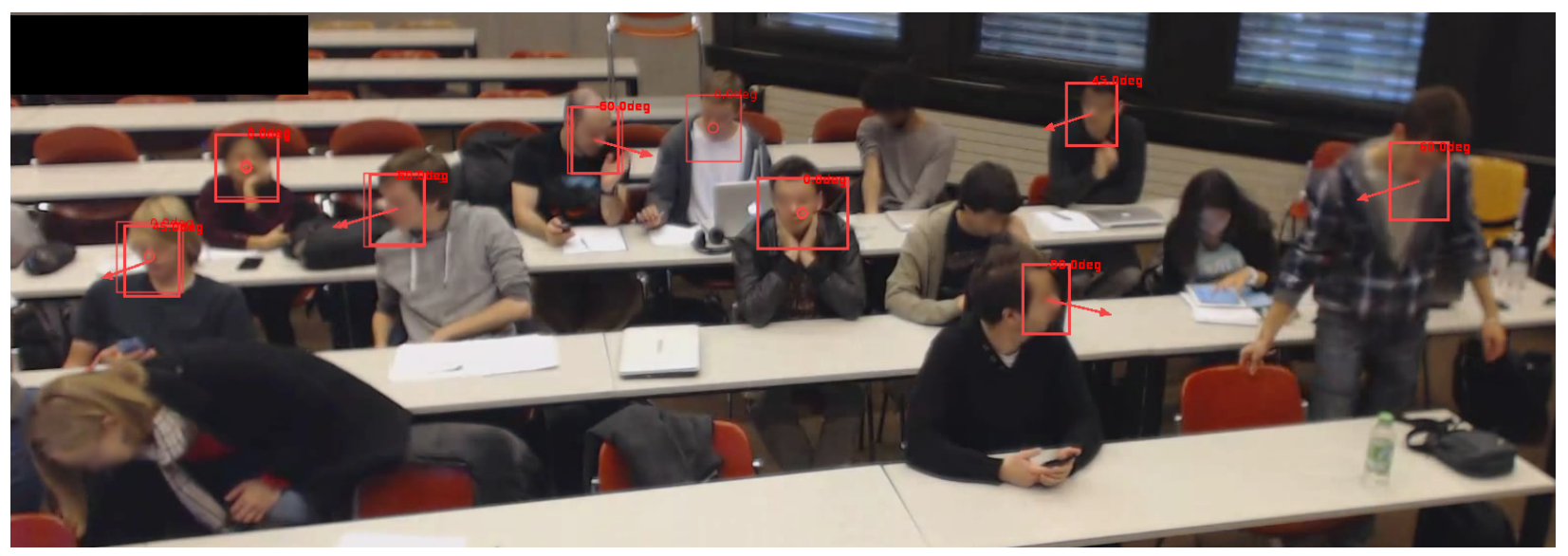

Figure 1: Captured footage of student activities in the classroom with estimates of the head orientation.

\begin{tabular}{|c|c|c|c|c|c|c|c|}
\hline Class & Size & Female ratio & Mean attendance & No. of sessions & No. of cameras & Post-class tests & Interviews \\
\hline 1 & 62 & $35.48 \%$ & $39.34(\sigma=1.15)$ & 3 & $5 / 1$ & 1 & 10 \\
\hline 2 & 43 & $34.88 \%$ & $27.5(\sigma=6.55)$ & 4 & $4 / 1$ & 3 & 0 \\
\hline
\end{tabular}

Table 1: Demographic statistics of the two analysed classes. Number of cameras shows the number of cameras capturing the student population and number of cameras capturing teacher's movement. In cases where we did not conduct the post-class tests, in-class tests were carried out.

classes, four sessions were conducted in this way. In one session we also tried using the clickers [6] but despite previously receiving usage instructions, students were slower to answer the questions and devices caused larger commotion in the student population than acceptable to the teacher.

Even though the teachers did not express dissatisfaction with the conduct (the filling out of the questionnaire lasted less than 4 minutes), we were concerned that our interruptions of the class could be changing students' perceptions of the lesson[1]. For this reason, we constructed another questionnaire (shown in Figure 7), which was meant to be filled out in two steps - before and after class, leaving the teaching period intact. The major changes were:

- in the part meant to be filled out before class we introduced a pre-test. In order to avoid priming of subjects [27], the pre-test was simulated by usage of two Likert scales capturing $i$ )interest and ii) knowledgability of the students without specifying the topics.

- Post-class part of the questionnaire captured the same four parameters as the in-class questionnaire, with visual aids (thumbnails of slides) introduced in order to give the students a better time reference.

To give the students more freedom when filling out the questionnaire, the Likert scales were replaced by areas in which they could draw out a "graph" to represent how they felt during the class about a certain parameter (we used the same 1-10 scale when transcribing the feedback). We also introduced gray-shadings to code the low/medium/high areas visually.

All gathered information was further processed as a longitudinal study. Each participant received an unique identifier which was kept consistent over the course of the study. With this effort we were also able to collect student seating customs and social connections (usual neighbors).

\subsection{Interviews}

We carried out a total of 10 individual interview sessions with the students. Due to time and budget restrictions, the interviews were done with the student from only a single class (Class 1). Our intention was to complement the quantitative nature of the questionnaires with more qualitative depth and to get feedback about the experiment and setup. The interviews were semi-structured, with open-ended answers, and were carried out the day after the experiment. An typical interview lasted (depending on the participant) between 15-30 minutes. Topics that were analyzed were i)perception of the environment (other students and outside events), ii)perception of the teacher, iii)students own preparation and mental state prior to the class, iv)seating habits, v)personal observations about their own behaviour vi)intrusiveness of the experiment. The interviews were recorded, and later analysed per category.

\section{OBSERVATIONS}

Our experiment resulted in a plentiful of data, which we continue to analyse. We'll present our preliminary findings about teacher's motion, student's perception of the experiment and challenges for further data interpretations.

\subsection{From the videos}

The coverage of the classroom with the array of webcameras has proven to be effective. We were able to effectively capture the students head orientation up to the distance of 4 meters away from the blackboard. In order to achieve this, we had to take on the effort of re-training a specialized face detector and to develop additional testing data-sets.

Under ideal conditions, most salient passive source of information about students interest during the class would be a set of eye-trackers. This would provide a significant source 


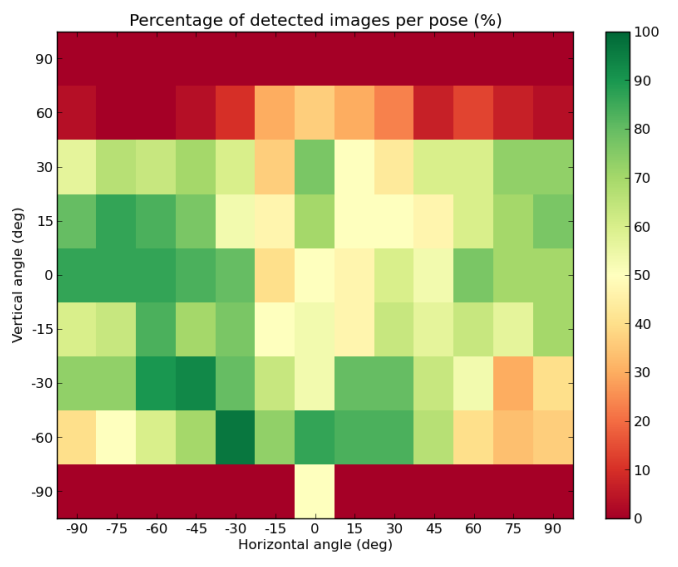

Figure 3: Coverage of detections on the Pointing'04 test-set of the face detector.

of data, under considerable restrictions in portability of the system and budget requirements. In order to go around the problem, our system relied on gaze estimation from the head orientation, which, in stead of analysing the psychological level of the individual, is stationed in the domain of sociological studies of "social gaze" [15], although limiting the resolution of data we can achieve from any individual.

Our detector/orientation estimator was able to cover a wide range of poses (pitch and yaw angles) of human head. On the Pointing'04 [17] which we used as a test-set, we achieved more than $50 \%$ coverage for all but extreme head angles (Fig. 3). We are still in the process of analysing the patterns from the student camera streams.

From the cameras observing teachers' motion, by using the TLD tracker/detector [18] we were able to track teacher's motion over the course of the entire class. We can visualize the $1 \mathrm{D}$ horizontal location of the teacher in front of the blackboard over time as shown in Figure 4. By using simple method of thresholding the variance of the neighbouring points, we managed to create a classification between standing and moving detection of the teacher. For the two teacher (with 2 analysed lectures for each teacher) we extracted the average moving/standing ratio to $50.82 \%$ and $23.27 \%$.

In order to make the motion of the teacher's comparable, given different room geometries, we normalized the location to a $0.0-1.0$ scale which represents the area in front of the blackboard. In order not to exclude the locations outside of this area, we did not clamp the values strictly to these values. From there we were able to extract information about the teacher's usage of space which we visualize in Figure 5. It's interesting to observe the tendency of standing on the sides of the blackboard, where the teacher does not obstruct the view of the slides/blackboard for the students. It also indicates that the teacher does not represent the central role in the classroom, but a role of guide in the learning process.

\subsection{From the questionnaires}

Our first test was to compare if there is a significant difference between the answers in two different questionnaire formats (in-class and post-class), in order to be able to use them interchangeably in further analysis. Our initial hypothesis was that the post-class format will display higher

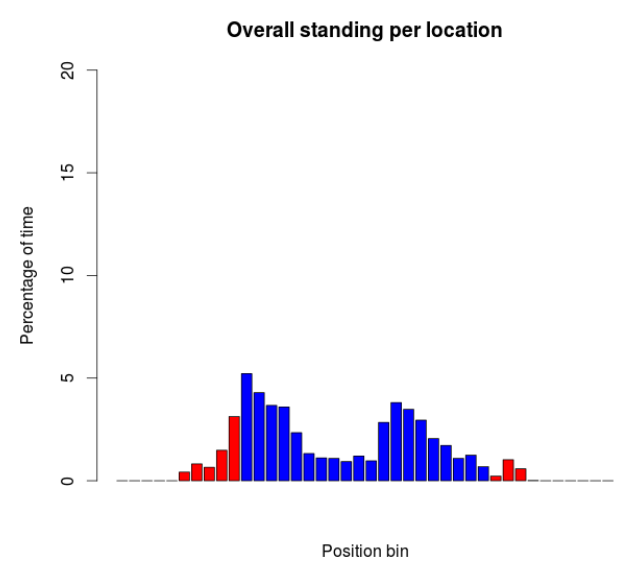

Figure 5: Average percent of time teacher's spent standing in each of the location bins. Blue bins represent the normalized area in front of the blackboard (scale 0.0-1.0), and the red bins represent the area outside of this range.

variance in the earlier periods of the class, given that they are further away from the questionnaire fill-out time.

The ANOVA tests found no significant difference over the aggregated values for $i$ )"attention" and $i i$ )"class attention" measurements, but significant difference in the iii)"material importance" $(f=6.662, p=0.01)$ and $i v)$ "teacher energy" $(f=19.557, p<0.01)$.

Further analysis of the two values which showed that the difference became more pronounced in the later periods of the class in both cases (Fig.6). In case of teacher energy, the difference was present starting from the second period ( 2 nd period $f=11.319, p<0.01$; 3rd period $f=25.62, p<0.01$; 4 th period $f=8.98, p<0.01)$. In case of the material importance the difference was present in the $\operatorname{3rd}(f=10.68$, $p<0.01)$ and 4 th period $(f=7.25, p<0.01)$. In both cases, students gave higher values in case of the post-class questionnaire.
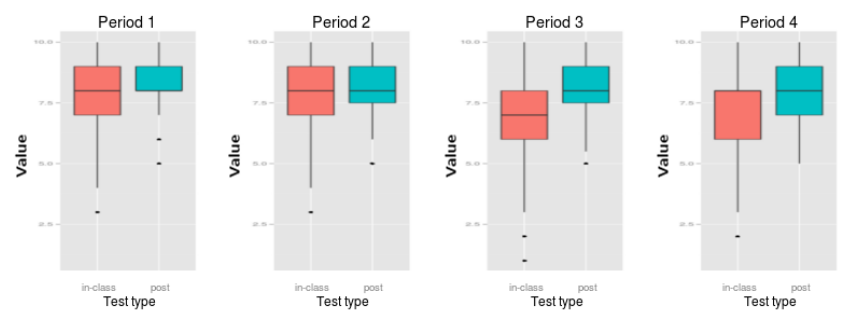

Figure 6: Difference between in-class (red) and post-class (teal) questionnaires for the measurement "teacher energy" at different periods. ANOVA tests shows the difference in periods 2,3 and 4 with $p<0.05$.

\subsection{From the interviews}

The interviews served as a way of gathering further qualitative information about the experiment. We conducted 10 interviews in total over the semester (female ratio 40\%). 


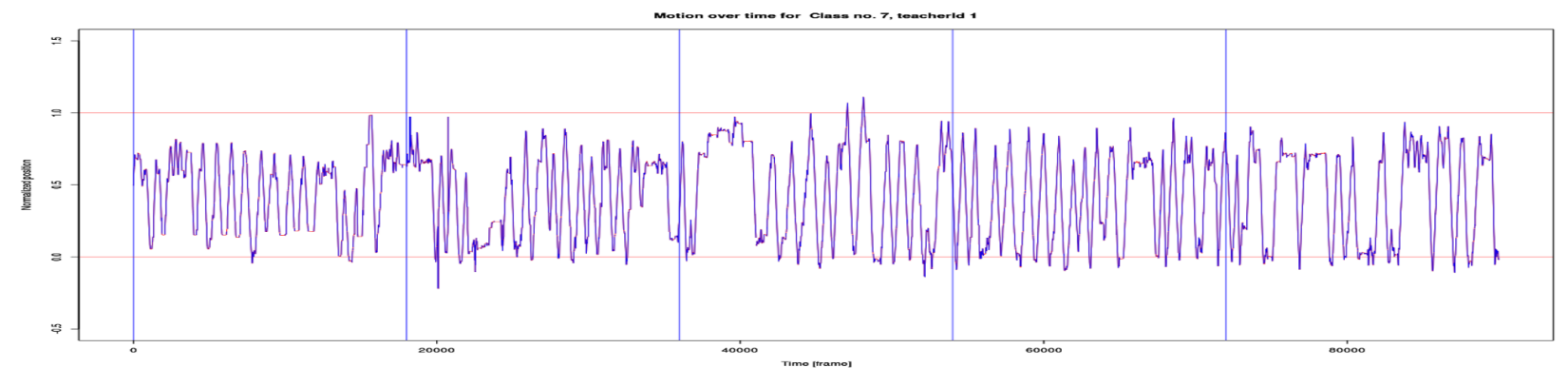

Figure 4: Teacher motion over time. Horizontal red lines represent the normalized area in front of the blackboard. Vertical blue lines represent 10-minute periods. We classified each point as standing (red) or moving (blue).

We first explored the disruption of the experiment on the classroom life. $80 \%$ of the subjects confirmed that they notice the experiment, but only $10 \%$ said that the experiment setup is overall disruptive. $60 \%$ agreed that the in-class format is disruptive (either to the positive or negative effect of their studying). Also, at least 2 participants said that the in-class questionnaires, although disruptive, were easier to answer and "more objective" from their point of view. We recorded no positive comments for the post-class questionnaire.

Further, we asked the subjects to describe different aspects of the lecture in order to see which modality was dominant in their perception of the classroom. Majority of the descriptions touched on the overall structure or feeling of the lecture (19 instances), and after that visual references (10 instances) were more present than auditory descriptions (7 instances). The point of the visual focus was mostly the slides of the lecture ( $80 \%$ of participants), and then the professor (60\% of participants).

In the description of their surroundings, only 1 subject described the ambient of the classroom (in positive or negative context). When questioned about their neighbours, $90 \%$ described their immediate neighbours, $30 \%$ could note some action or detail about their neighbours in front, and $20 \%$ about persons sitting behind them.

\section{DISCUSSION}

Our first goal is the visual attention - where the students are looking during the lecture and what that indicates to other students. We have already seen studies in controlled environments that people are following other people's direction of gaze to gather information what is important to observe in a new scene [5]. We can benefit from the implicit constraints of the classroom environment to differentiate between classroom engagement (learner-instructor), material engagement (learner-content) or interactions between students (learner-learner) [21]. Apart from this formal division which we can use to determine the dominant source of information at any given time, it's more interesting to observe when do the unintentional actions of other students become observed by their neighbours.

That leads us to the second potential benefit - segmenting the classroom population. We would like to see whether our measurements can be better at grouping people into study-groups or re-arranging the classroom into a more engaged configuration. We already know that the position of the students in the classroom can modify their engagement, front-center being most likely to engage into discussion, and back-edge being least engaged [2]. If we can differentiate between engagement/disengagement periods in class, a system could recommend groups of students who's attention covered different parts of the class in order to stimulate better discussions.

Switching over to combinations of observations, correlating teacher's actions with the reaction of the students can be very beneficial for identifying good approaches in classroom orchestration. Also, it could indicate to the teacher which part of the classroom was less active and stimulate the teachers in distributing their attention equally among the population. The beginning of motion analysis of the teacher identified a predictable pattern of standing, which confirms the role of teacher as a guide, and not a "sage on a stage". It would be interesting to compare different "motion signatures" between different teachers and determine if there is a pattern beyond geometrical constraints of the room, as indicated by earlier work in spatial pedagogy [19].

We are still developing the means to process the teacher's gaze captured by the eye-trackers. Even though wearing eyetrackers during the class is not an input we can expect from every classroom, and therefore it can not be a feature we depend on, it can shed light on the teacher's perception of the classroom. Further work is needed in order to stabilize and orient the picture received from the mobile eye-tracker.

For the moment we are not processing the sound modality of the classroom, for the reason that we are more interested on how students are behaving when they are not in the direct interaction with teacher (assuming that the teacher is controlling the classroom, we expect the students to be silent for a better part of the class). This does not mean that the sound is irrelevant in the classroom, and we already know that visualizing sound interaction can bring significant insight in the meetings [4], and it would further help us gather additional information how the teacher can "reach out" to the students. We hypothesize that the sound direction/speaker classification would be a reliable way of classifying QA periods of the class, the speed of response of the students and finding how the interactivity of the class changed over time.

The observation and quantification of gaze is well within the "observational principle" [29] which governs our inquiry. By comparing the observed patterns of behaviour to the questionnaire data, we work towards modelling the subjec- 
tive view of the students about their attention. Another challenge would be to introduce the EEG (electroencephalography) measurements as the biological basis for both. Brain activity would be the most objective measurement we can achieve in the experiment, and the price accessibility of the consumer-level EEG devices puts the intervention in the realm of possible. But it does not come without risk. The high intrusiveness of such an intervention needs to be carefully organized, and technical limitations of the low-end devices limits the quality of experiments which can be run outside of a controlled environment.

\section{CONCLUSION}

It is difficult to determine what makes a good class. The practical answer is the score the students achieve in the test at the end of semester - which encapsulates students interests, home-studying, classroom engagement and in some cases proficiency to cheat. Capturing the classroom dynamics makes it possible to analyse what goes on between the students and the teacher, but up until now it has been considered impractical, if not impossible. Only with the development with new systems for automatic capturing and processing of the learning activity can we gain insight into the meaningful pieces of information from the vast quantity of captured material. In this sense, even with a population of mere 50 students, every class is a source of "big data".

Analysis of the teaching/learning process does not mean necessary evaluation of the student or the teacher. We do not think that the only benefit that can come out of the observations needs to be quantified on the scale from 110, but rather to help professors identify the good practices in their teaching. With the diversity of subjects, teachers and students, the combination space quickly becomes too big for a single solution, and for this reason, we question the idea of finding "the best practice". But we do hope to encourage teachers to find their own best practice, and we aim to provide them with tools which will aid them in this search.
We have presented our efforts into capturing a complete picture of the classroom experience. In this paper we focused on the potential results we can achieve with our videomonitoring system, and the extent of information we can extract with the currently available algorithms. We complemented the observations with additional textual inputs from the students and in-depth interviews about students' perception of the classroom, lecture and experiment. We validated that the system is acceptable to the student population, which encourages the thought that the solutions developed for the project will find their way into the real classrooms and not just experiment set-ups.

Social research is always dependent on the choice of how to make the scientific cut between the measurement we want, and outside influences we can not control. With every new technological development we aim to push the boundary of what we can observe a little further towards a complete representation of the human interaction. By further developing quantifiable approaches, we hope that the trade-off that the experiments have to make will diminish over time, and give us bigger samples from realistic situations.

\section{ACKNOWLEDGMENTS}

We would like to thank all professors and students who participated in our experiments, as well as Jessica Dahler for her input and help with the design of the questionnaire. The authors would also like to thank Luis Pablo Prieto for his comments and suggestions about the text, and Hilton Bristow for his work on open implementation of part based model detector which we used for face detection. This work was sponsored by the ProDoc SNF Grant, project PDFMP1 135108. 


\section{REFERENCES}

[1] J. G. Adair. The hawthorne effect: A reconsideration of the methodological artifact. Journal of applied psychology, 69(2):334, 1984.

[2] R. S. Adams. Location as a feature of instructional interaction. Merrill-Palmer Quarterly of Behavior and Development, 15(4):309-321, 1969.

[3] I. Arroyo, D. G. Cooper, W. Burleson, B. P. Woolf, K. Muldner, and R. Christopherson. Emotion sensors go to school. In AIED, volume 200, pages 17-24, 2009.

[4] K. Bachour. Augmenting face-to-face collaboration with low-resolution semi-ambient feedback. $\mathrm{PhD}$ thesis, ÉCOLE POLYTECHNIQUE FÉDÉRALE DE LAUSANNE, 2010.

[5] E. Birmingham, W. F. Bischof, and A. Kingstone. Social attention and real-world scenes: The roles of action, competition and social content. The Quarterly Journal of Experimental Psychology, 61(7):986-998, 2008.

[6] J. E. Caldwell. Clickers in the large classroom: current research and best-practice tips. CBE-Life Sciences Education, 6(1):9-20, 2007.

[7] D. Clow. Moocs and the funnel of participation. In Proceedings of the Third International Conference on Learning Analytics and Knowledge, pages 185-189. ACM, 2013.

[8] D. Conigliaro, F. Setti, C. Bassetti, R. Ferrario, and M. Cristani. Attento: Attention observed for automated spectator crowd analysis. In Human Behavior Understanding, pages 102-111. Springer, 2013.

[9] M. Csikszentmihalyi and M. Csikzentmihaly. Flow: The psychology of optimal experience, volume 41. HarperPerennial New York, 1991.

[10] J. A. Daly and A. Suite. Classroom seating choice and teacher perceptions of students. The Journal of Experimental Educational, pages 64-69, 1981.

[11] J. Daniel. Making sense of moocs: Musings in a maze of myth, paradox and possibility. Journal of Interactive Media in Education, 3, 2012.

[12] P. Dillenbourg and P. Jermann. Technology for classroom orchestration. New Science of Learning, pages 525-552, 2010.

[13] P. Dillenbourg, G. Zufferey, H. Alavi, P. Jermann, S. Do-Lenhand, Q. Bonnard, S. Cuendet, and F. Kaplan. Classroom orchestration: The third circle of usability. In International Conference on Computer Supported Collaborative Learning Proceedings, pages 510-517. 9th International Conference on Computer Supported Collaborative Learning, 2011.

[14] R. El Kaliouby and P. Robinson. Mind reading machines: Automated inference of cognitive mental states from video. In Systems, Man and Cybernetics, 2004 IEEE International Conference on, volume 1, pages 682-688. IEEE, 2004.

[15] N. Emery. The eyes have it: the neuroethology, function and evolution of social gaze. Neuroscience $\mathcal{E}$ Biobehavioral Reviews, 24(6):581-604, 2000.

[16] T. Fenwick, M. Nerland, and K. Jensen. Sociomaterial approaches to conceptualising professional learning and practice. Journal of Education and Work, 25(1):1-13, 2012.
[17] N. Gourier, D. Hall, and J. L. Crowley. Estimating face orientation from robust detection of salient facial structures. In FG Net Workshop on Visual Observation of Deictic Gestures, pages 1-9. FGnet (IST-2000-26434) Cambridge, UK, 2004.

[18] Z. Kalal, K. Mikolajczyk, and J. Matas. Tracking-learning-detection. Pattern Analysis and Machine Intelligence, IEEE Transactions on, 34(7):1409-1422, 2012.

[19] F. Lim, K. O'Halloran, and A. Podlasov. Spatial pedagogy: mapping meanings in the use of classroom space. Cambridge Journal of Education, 42(2):235-251, 2012.

[20] A. Mazzei, T. Koll, F. Kaplan, and P. Dillenbourg. Attentional processes in natural reading: the effect of margin annotations on reading behaviour and comprehension. In Proceedings of the Symposium on Eye Tracking Research and Applications, pages 235-238. ACM, 2014.

[21] M. G. Moore. Editorial: Three types of interaction. 1989.

[22] M. Raca and P. Dillenbourg. System for assessing classroom attention. In Proceedings of the Third International Conference on Learning Analytics and Knowledge, pages 265-269. ACM, 2013.

[23] M. Raca, R. Tormey, and P. Dillenbourg. Sleepers' lag-study on motion and attention. In Proceedins of the Fourth International Conference on Learning Analytics And Knowledge, pages 36-43. ACM, 2014.

[24] V. Rivera-Pelayo, J. Munk, V. Zacharias, and S. Braun. Live interest meter: learning from quantified feedback in mass lectures. In Proceedings of the Third International Conference on Learning Analytics and Knowledge, pages 23-27. ACM, 2013.

[25] D. Schon. The reflective practitioner: How professionals think in action, volume 5126. Basic Books, 1984.

[26] R. Stiefelhagen and J. Zhu. Head orientation and gaze direction in meetings. In CHI'O2 Extended Abstracts on Human Factors in Computing Systems, pages 858-859. ACM, 2002.

[27] E. Tulving and D. L. Schacter. Priming and human memory systems. Science, 247(4940):301-306, 1990.

[28] W. Wang, V. Enescu, and H. Sahli. Towards real-time continuous emotion recognition from body movements. In Human Behavior Understanding, pages 235-245. Springer, 2013.

[29] E. J. Webb, D. T. Campbell, R. D. Schwartz, and L. Sechrest. Unobtrusive measures, volume 2. SAGE Publications, Incorporated, 1999. 
Class questionnaire

\section{Pre-class questions}

Date:

Seating location (row, seat):

How interested are you in todays topic?

How knowledgable are you about the topic? \begin{tabular}{|l|l|l|l|l|l|l|l|l|l|}
1 & 2 & 3 & 4 & 5 & 6 & 7 & 8 & 9 & 10 \\
\hline
\end{tabular}

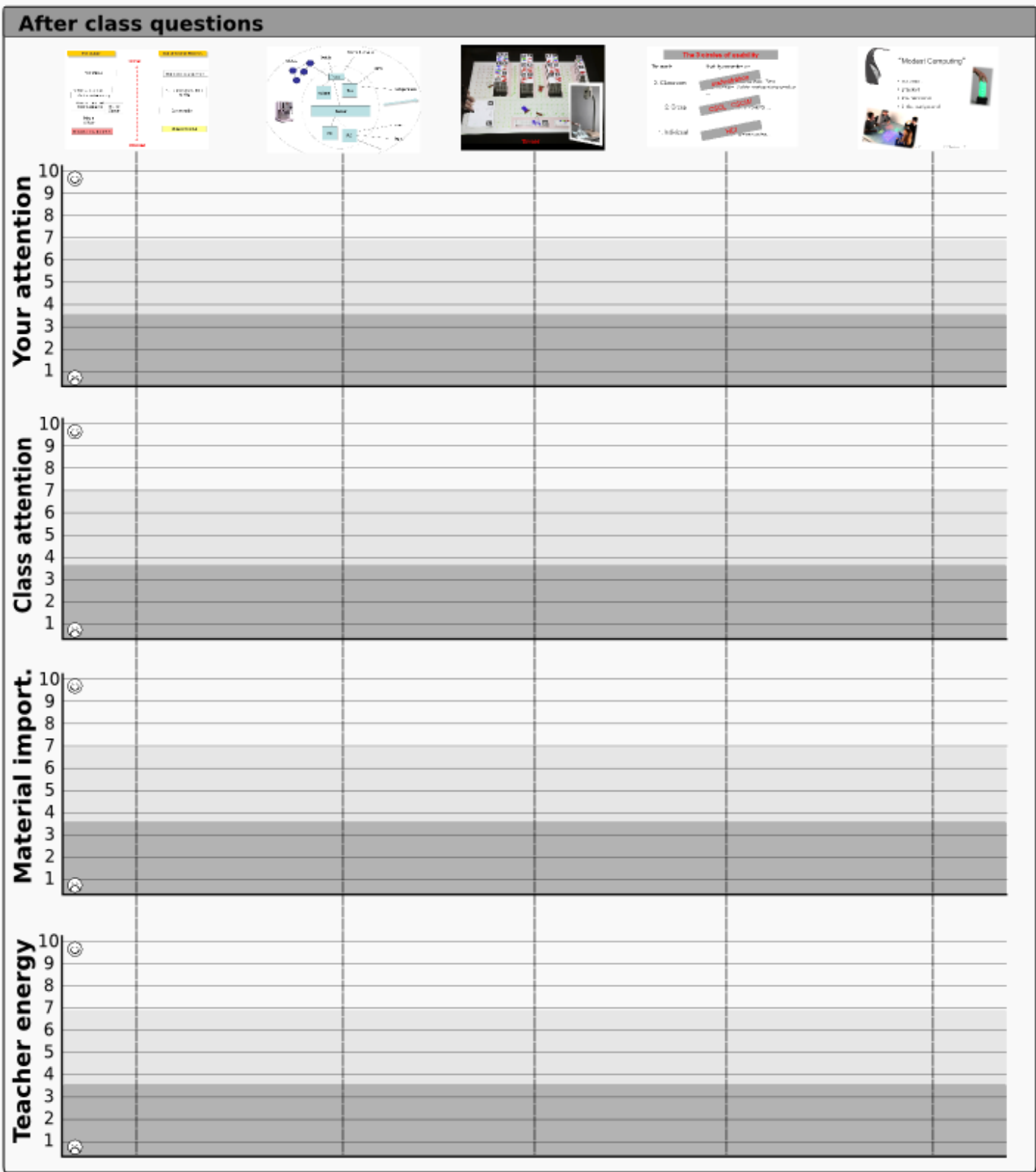

t

Figure 7: Example of the post-class format of the questionnaire. In the pre-class period we approximated a pre-test without priming the students with questions about $i$ ) how interested and ii) how knowledgeable are they about the subject. In the post-class section we used lecture slides to convey time of the class, and color-gradient for each question to demonstrate low-middle-high regions. 